\title{
Non-rigid Image Registration using Electric Current Flow
}

\author{
Shu Liao, Max W. K. Law and Albert C. S. Chung \\ Lo Kwee-Seong Medical Image Analysis Laboratory, \\ Department of Computer Science and Engineering, \\ The Hong Kong University of Science and Technology, Hong Kong \\ $\{1$ iaoshu, maxlawwk, achung\}@cse.ust.hk
}

\begin{abstract}
In this paper, a novel electric current flow (ECF) based model is proposed to perform feature based non-rigid brain image registration. The ECF features simultaneously capture both voxel intensity and inter-voxel distance information. In the proposed ECF framework, each voxel is regarded as exhibiting electric potential proportional to voxel intensity. Voxels are connected by conductive wires in a pairwise manner. Each conductive wire has resistance, in which the resistance value is proportional to the length of the wire. The electric potential difference among connected pixels induces electric current passing through their connected wire. The amount of the electric current is the ratio between the voxel potential difference and the wire resistance. The potential difference and resistance are respectively proportional to the voxel intensity difference and the inter-voxel distance. By analyzing the electric current induced by the connection between a reference voxel and its counterparts in a given range, the ECF algorithm searches for the most salient connection to construct the ECF features. The ECF features are incorporated in the Markov random field labeling framework for non-rigid image registration. The registration quality of the proposed method has been evaluated intensively on both BrainWeb and IBSR databases. It is compared with four related approaches. Experimental results illustrate that the proposed method consistently achieves the highest registration accuracy among all the compared methods on both databases.
\end{abstract}

\section{Introduction}

A variety of non-rigid image registration approaches have been developed for medical image analysis. Intensity based approaches have widely been used for registering template and subject images based on inter-image intensity similarity estimated from image joint intensity distributions. However, intensity similarity does not necessarily equivalent to anatomical similarity. As pointed out in [9], the mere use of intensity information is possibly ineffective to distinguish between different brain anatomical structures. On the other hand, feature based registration methods use feature vector as anatomical signature for each voxel, where the registration problem is formulated as a feature matching problem. Observing intensity or gradient similarity between subject and template images are common criteria for finding correspondences [4]. Apart from voxel intensity and local image gradient, as will be demonstrated in this paper, it is beneficial to consider inter-voxel relation between distant voxels rather than relation between adjacent voxels. It is because the distant voxel relation offers additional intensity and geometric information within a predefined region of a voxel. A new feature, namely Electric Current Flow (ECF), is proposed for representing both the geometric information (inter-voxel distance) and intensity information (voxel intensity difference) at various scales. The ECF feature is integrated with the Markov random field deformation model to perform non-rigid image registration. It is evaluated on two publicly available databases - BrainWeb and IBSR. The proposed method is compared with four state-of-the-art registration approaches.

\section{The electric current flow (ECF) feature}

Each pair of voxels, denoted as $\overrightarrow{v_{1}}$ and $\overrightarrow{v_{2}}$, is assumed to be connected by a conductive wire. At each voxel position, it is assumed that its electric potential is represented by the its intensity, denoted as $G\left(\overrightarrow{v_{1}}\right)$ and $G\left(\overrightarrow{v_{2}}\right)$, respectively. The relationship between $\overrightarrow{v_{1}}$ and $\overrightarrow{v_{2}}$ is established based on the Ohm's law. The voltage between $\overrightarrow{v_{1}}$ and $\overrightarrow{v_{2}}$ is therefore the absolute value of the potential difference between them, which is expressed as $V\left(\overrightarrow{v_{1}}, \overrightarrow{v_{2}}\right)=\left|G\left(\overrightarrow{v_{1}}\right)-G\left(\overrightarrow{v_{2}}\right)\right|$. The resistance between $\overrightarrow{v_{1}}$ and $\overrightarrow{v_{2}}$ is modeled by their voxel distance, which is given by $R\left(\overrightarrow{v_{1}}, \overrightarrow{v_{2}}\right)=\left|\overrightarrow{v_{1}}-\overrightarrow{v_{2}}\right|$. It is therefore assumed that the resistance increases proportional to the distance between two voxels. Such assumption reflects the physical phenomenon that the longer the wire, the larger the resistance. Then, the amount of current passing through $\overrightarrow{v_{1}}$ and $\overrightarrow{v_{2}}$ is obtained by dividing the voltage value 
by the resistance according to the Ohm's law which is given as

$$
I\left(\overrightarrow{v_{1}}, \overrightarrow{v_{2}}\right)=\frac{V\left(\overrightarrow{v_{1}}, \overrightarrow{v_{2}}\right)}{R\left(\overrightarrow{v_{1}}, \overrightarrow{v_{2}}\right)}=\frac{\left|G\left(\overrightarrow{v_{1}}\right)-G\left(\overrightarrow{v_{2}}\right)\right|}{\left|\overrightarrow{v_{1}}-\overrightarrow{v_{2}}\right|}
$$

In this paper, it is assumed that the larger the amount of current passing through $\overrightarrow{v_{1}}$ and $\overrightarrow{v_{2}}$, the more salient the anatomical information encoded in the inter-voxel relationship between $\overrightarrow{v_{1}}$ and $\overrightarrow{v_{2}}$. For a reference voxel $\vec{v}$, voxel $\vec{v}$ is called the ECF source point of $\vec{v}$ if the amount of current calculated by Equation 1 passing through $\vec{v}$ and $\vec{v}$ is maximized with respect to $\vec{v}$. The ECF source point $\vec{v}$ of $\vec{v}$ can be expressed by Equation 2,

$$
\overrightarrow{\vec{v}}=\arg \max _{\overrightarrow{v^{\prime}} \in \Omega} I\left(\overrightarrow{v^{\prime}}, \vec{v}\right)
$$

where $\Omega$ represents the image domain. The amount of current passing through $\vec{v}$ and $\overrightarrow{\vec{v}}$ is used as the voxel signature for $\vec{v}$, which is defined by Equation 3

$$
\operatorname{ECF}(\vec{v})=I(\overrightarrow{\vec{v}}, \vec{v})
$$

where $\vec{v}$ is the ECF source point of $\vec{v}$ calculated by Equation 2 .

As pointed out in [10], feature saliency is closely related to the scale from which it is extracted. Extracting features from only a single scale of interest generally is insufficient to capture all the anatomical information around the reference voxel. To extract the ECF features from different scales, the search domains for finding the ECF source points in Equation 2 are confined in spheres centered at $\vec{v}$ with different values of radius $r$ instead of the whole image domain, denoted as $\Delta_{r}^{\vec{v}}$, where $r$ reflects the scale of interest. Therefore, now the ECF signature of $\vec{v}$ with scale of interest $r$ is defined as,

$$
E C F(\vec{v}, r)=I\left(\overrightarrow{\hat{v_{r}}}, \vec{v}\right)=\frac{V\left(\overrightarrow{\hat{v}_{r}}, \vec{v}\right)}{R\left(\overrightarrow{\hat{v}_{r}}, \vec{v}\right)}
$$

where $\overrightarrow{v_{r}}$ is the ECF source point of $\vec{v}$ with scale of interest $r$, it is defined as:

$$
\overrightarrow{\hat{v}_{r}}=\arg \underset{\overrightarrow{v^{\prime} \in \Delta_{r}^{\vec{v}}}}{\max } I\left(\overrightarrow{v^{\prime}}, \vec{v}\right) .
$$

The ECF feature signature of each reference voxel is obtained by concatenating the ECF features calculated by Equation 4 from different scales $r$. Algorithm 1 summarizes the procedure of calculating the ECF feature signatured image $F$ with $k$ scales of interest. The ECF feature has several distinctive properties.

Property 1: The ECF feature is $3 D$ rotation invariant. It is given that the ECF feature is the maximum amount of current passing through the reference voxel and

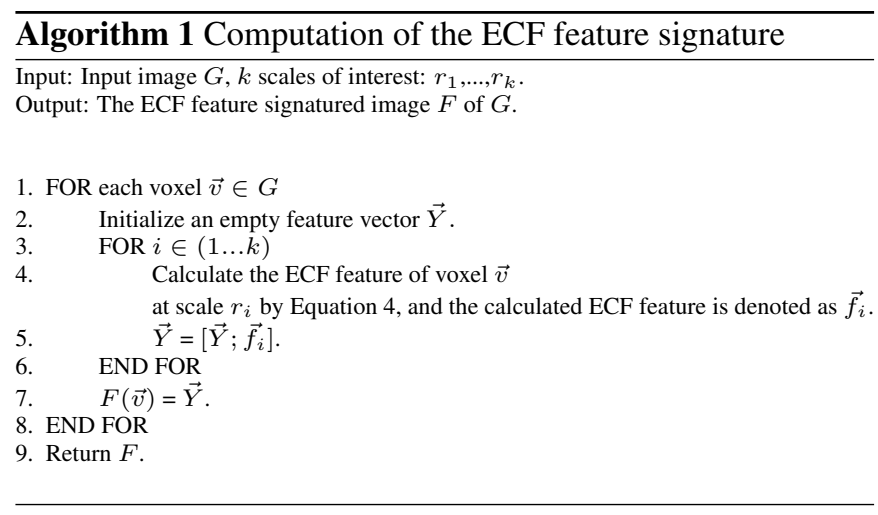

its corresponding ECF source point within a local spherical search domain, as stated in Eqns 4 and 5. Although the image is rotated, the ECF source point remains the same within such spherical search domain because the maximum current is still the same between the reference voxel and its original ECF source point. Thus the ECF feature remains the same, despite of image rotation.

Property 2: The conventional gradient magnitude feature is a special case of the ECF feature. It should be noted that the ECF source point is calculated based on Equation 5, where the ECF source point is found within a spherical search domain centered at the reference voxel with a specified scale of interest $r$ (i.e., the radius of the spherical search domain). When $r$ equals one, it means that the ECF source point is now only found within the immediate neighboring voxels with respect to the reference voxel. In this case the denominator of Equation 4 is equal to one, and the numerator of Equation 4 now specifies the largest intensity changes between the reference voxel and its immediate neighboring voxels. This is exactly the physical meaning of the conventional gradient magnitude feature.

Property 3: The maximum distance gradient magnitude (MDGM) feature [2] is a special case of the ECF feature. The MDGM feature is defined based on the distance gradient operator $\nabla_{d}$ between two arbitrary different voxels $\overrightarrow{v_{1}}$ and $\overrightarrow{v_{2}}$, which is defined as [2]: $\nabla_{d} G\left(\overrightarrow{v_{1}}, \overrightarrow{v_{2}}\right)=\left(G\left(\overrightarrow{v_{1}}\right)-G\left(\overrightarrow{v_{2}}\right)\right) \cdot \frac{\overrightarrow{v_{1}}-\overrightarrow{v_{2}}}{\left|\overrightarrow{v_{1}}-\overrightarrow{v_{2}}\right|^{2}}$, where $G$ denotes the input image, $G\left(\overrightarrow{v_{1}}\right)$ and $G\left(\overrightarrow{v_{2}}\right)$ denote the input image intensity values at positions $\overrightarrow{v_{1}}$ and $\overrightarrow{v_{2}}$, respectively. Then the MDGM feature for voxel $\vec{v}$ is defined as, $\operatorname{MDGM}(\vec{v})=\left|\nabla_{d} G(\vec{v}, \vec{v})\right|$, where $\overrightarrow{\vec{v}}$ is the MDGM source point of $\vec{v}$ defined as: $\overrightarrow{\vec{v}}=\arg \max _{\overrightarrow{v^{\prime}} \in \Omega}\left|\nabla_{d} G\left(\overrightarrow{v^{\prime}}, \vec{v}\right)\right|$, where $\Omega$ is the image domain of $G$. Therefore, the ECF feature becomes exactly the MDGM feature when the spherical search domain in Equation 5 to find the ECF source point is large enough to cover the whole image domain. 


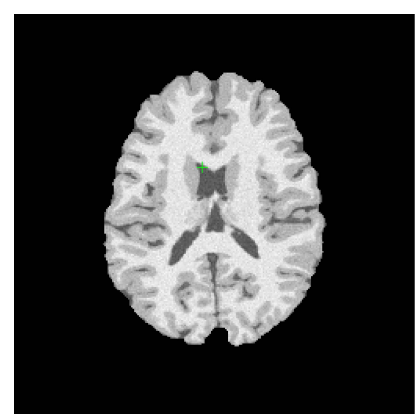

(a)

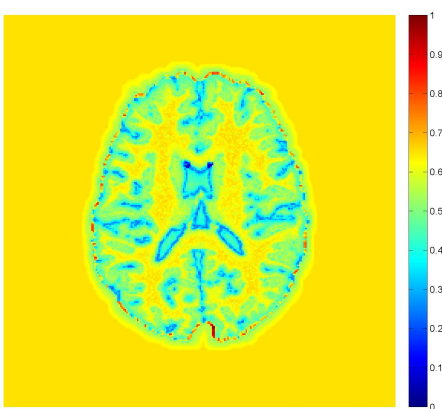

(b)

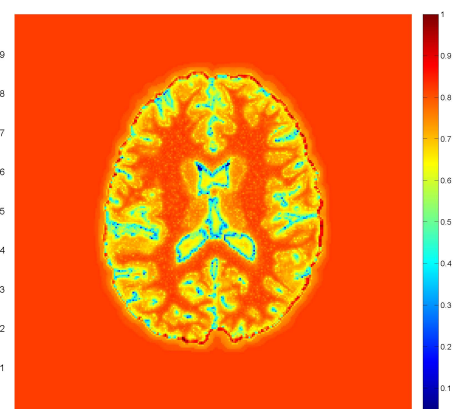

(c)

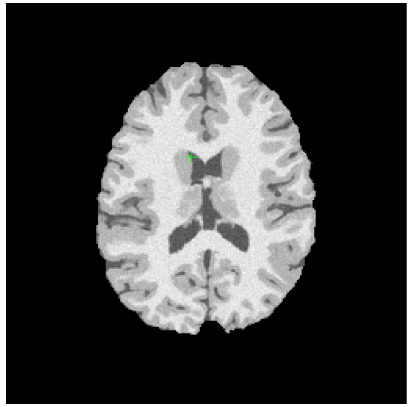

(d)

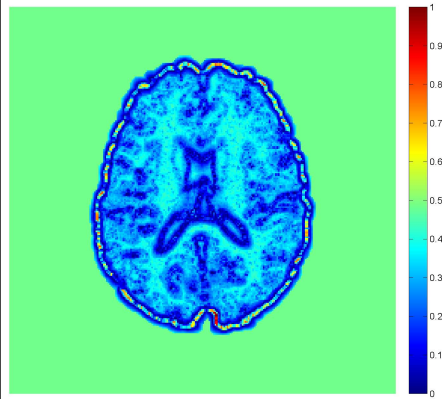

(e)

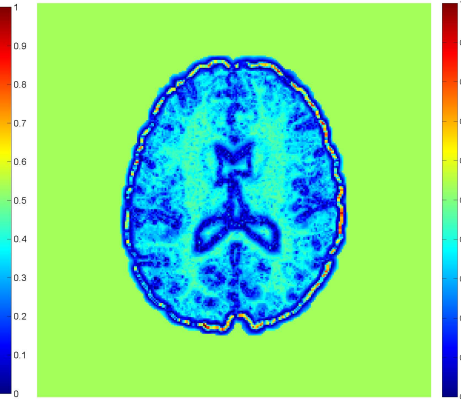

(f)

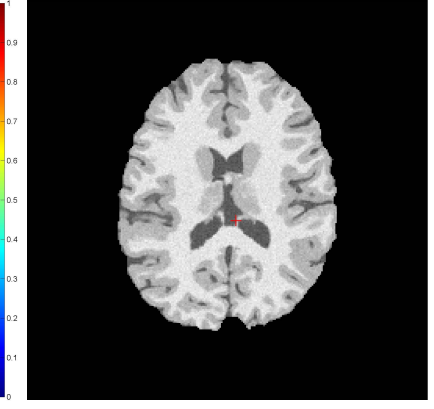

$(\mathrm{g})$

Figure 1. (a) An image obtained from BrainWeb, the features of the voxel highlighted with the green cross is compared with the features of other voxels; (b) The difference map of image shown in (a) using ECF, blue indicates high similarity; (c) The difference map obtained by comparing the ECF feature of the highlighted voxel in (a) with all the voxels in the image shown in (d); (d) An image obtained from BrainWeb with a different patient from (a). The green cross in (d) denotes the voxel in the image (d) with the most similar ECF feature compared to the highlighted voxel in (a); (e) and (f) are the intra-patient and inter-patient difference maps using MDGM [2] respectively. The red cross in $(\mathrm{g})$ denotes the voxel in the image (d) with the most similar conventional MDGM feature compared to the highlighted voxel in (a). Please refer to the electronic version of this paper for better visualization.

We now demonstrate the superior discriminant power of the ECF feature signature over the MDGM feature. Figure 1a is an MR image obtained from BrainWeb, and the green cross in Figure 1a denotes the referencing voxel $\overrightarrow{r e f}$. The ECF feature signature of this referencing voxel is compared with the ECF feature signatures of other voxels. Figure $1 \mathrm{~b}$ is the color-coded difference map of the image shown in Figure 1a with the ECF feature signatures calculated from three different scales $r_{1}=3$, $r_{2}=5, r_{3}=8$ for each voxel. Figure $1 \mathrm{~b}$ shows that (from the blue voxels) the ECF features of $\vec{v}_{\text {ref }}$ in Fig. 1a are only similar to a small number of voxels with similar anatomical properties with $\overrightarrow{v_{e f}}$ (i.e., near the ventricle corner). It reflects the discriminant power of ECF feature signature to identify different anatomical structures. Moreover, voxels of similar anatomical positions across different patients should have similar feature signatures for establishing reliable correspondences. The difference map between the ECF feature signatures of $\vec{v}_{r e f}$ in Figure $1 \mathrm{a}$ and all the voxels in the image shown in Figure $1 \mathrm{~d}$ is also calculated and shown in Figure 1c. The MR image shown in Figure 1d is also obtained from BrainWeb but with a different patient from the one shown in Figure 1a. It is observed that the ECF feature signatures of $\vec{v}_{\text {ref }}$ in Figure 1a are only similar to the voxels in Figure 1c which are also located near the ventricle corner. For the image shown in Figure 1d, the voxel with the most similar ECF feature signature compared to $\overrightarrow{v_{r e f}}$ in Figure 1a is also highlighted with a green cross. It is also located near the ventricle corner. Thus, the ECF feature signatures can also provide reliable correspondences between voxels with similar anatomical properties across two patients. The difference map generated by the MDGM feature [2] is also included for comparison. Figures $1 \mathrm{e}$ and $\mathrm{f}$ are the intra-patient and inter-patient difference maps obtained by the MDGM feature with the same referencing pixel in Figure 1a respectively, and lots of false similarity matches are identified. The voxel with the most similar MDGM feature of the image shown in Figure 1d compared to $\overrightarrow{v_{r e f}}$ in Figure 1a is highlighted with a red cross shown in Figure $1 \mathrm{~g}$, which is obviously located at a different anatomical position. Therefore, the higher discriminant power of ECF over MDGM is implied in this example. 


\section{Deformation Model for ECF}

In this paper, the ECF signatured image is integrated with the Markov random field (MRF) labeling framework $[3,7]$ to conduct registration. The general form of the MRF energy function is defined as,

$$
\begin{aligned}
E_{f} & =E_{\text {data }}+E_{\text {smoothness }} \\
& =\sum_{p \in \Omega} D_{p}\left(l_{p}\right)+\sum_{(p, q) \in N} V_{p, q}\left(l_{p}, l_{q}\right),
\end{aligned}
$$

where $\Omega$ is the image domain, and $N$ is the neighborhood system. The 6-connected neighborhood system is used in this paper. $D_{p}\left(l_{p}\right)$ denotes the cost function of the data term. It represents the cost of assigning label $l_{p}$ to voxel p. $V_{p, q}\left(l_{p}, l_{q}\right)$ is the cost function of the smoothness term, and it penalizes the cost of label discrepancy between two neighboring voxels. The deformation space is quantized into a discrete set of labels $L \in\left\{l^{1}, l^{2}, \ldots, l^{n}\right\}$. Each label $l^{i}(1 \leq i \leq n)$ corresponds to a displacement vector $\vec{d}_{i}$. Labeling the voxel $p$ with label $l_{p}$ denotes moving $p$ to a new position by the displacement vector $\overrightarrow{d_{l_{p}}}$. The quantization step described in [7] is adopted in this paper, where each voxel can move from the original position to a new position bounded by a discretized window $\Psi=\{0, \pm s, \pm 2 s, \ldots, \pm w s\}^{d}$ with dimension $d$. In this paper, we set $s=1$. The cost function $D_{p}\left(l_{p}\right)$ of the data term is defined based on the ECF features:

$$
\begin{aligned}
D_{p}\left(l_{p}\right) & =D_{p}\left(G_{\text {template }}(\vec{p}), G_{\text {subject }}\left(\vec{p}+\overrightarrow{d_{l_{p}}}\right)\right. \\
& =D_{p}\left(\vec{F}_{\text {template }}(\vec{p}), \vec{F}_{\text {subject }}\left(\vec{p}+\overrightarrow{d_{l_{p}}}\right)\right) \\
& =\left\|\vec{F}_{\text {template }}(\vec{p})-\vec{F}_{\text {subject }}\left(\vec{p}+\overrightarrow{d_{l_{p}}}\right)\right\|_{L_{2}},(7)
\end{aligned}
$$

where $G_{\text {template }}$ and $G_{\text {subject }}$ denote the template and subject images, $\vec{F}_{\text {template }}$ and $\vec{F}_{\text {subject }}$ are the ECF feature signature images of the template and subject images respectively calculated from different scales of interest by Algorithm 1 at the current iteration. $\|\cdot\|_{L_{2}}$ denotes the $L_{2}$ distance. The piece-wise truncated absolute distance is used as the smoothness cost function given by $V_{p, q}\left(l_{p}, l_{q}\right)=$ $\min \left(\lambda,\left|\overrightarrow{d_{l_{p}}}-\overrightarrow{d_{l_{q}}}\right|\right)$, where $\lambda$ is a constant representing the maximum penalty, and it was set to 20 in this paper. The $\alpha$-expansion algorithm [11] is used to minimize the energy function defined in Equation 6 in this paper and it guarantees that the solution is within a known constant range of the global minimum.

\section{Experimental Results}

We have evaluated the proposed method on both the simulated and real 3D databases obtained from BrainWeb ${ }^{1}$

\footnotetext{
${ }^{1}$ http://www.bic.mni.mcgill.ca/brainweb/
}

and IBSR ${ }^{2}$, respectively. The proposed method was compared with three widely used methods: FFD [5], Demons [8] and HAMMER [6]. For comparison purpose, the results obtained by using the MDGM feature [2] with the same MRF based deformation model are included. In all the experiments, five different scales were used for the ECF features: $r_{1}=3, r_{2}=5, r_{3}=8, r_{4}=10, r_{5}=12$. The 3D displacement window used in this paper was $\Psi=$ $\{0, \pm 1, \pm 2, \ldots, \pm 12\}^{3}$. The control point spacing for FFD [5] was set to $2.5 \mathrm{~mm}$ as suggested in [1]. Each image was preprocessed by a skull stripping process using the Brainsuite software ${ }^{3}$ as it is a required step for the HAMMER [6] method compared in this paper. Affine registrations were first applied to register the subject images to the template image before conducting the non-rigid image registration. All the experiments were run on a $3 \mathrm{GHz}$ duo core CPU, 2GB RAM computer. The FFD and Demons algorithms were implemented based on ITK ${ }^{4}$. For HAMMER, its software provided by the authors of HAMMER was adopted ${ }^{5}$. The proposed method was also implemented based on ITK.

For the BrainWeb dataset, all twenty brain MR images were obtained. Each image has volume size of $256 \times 256 \times$ 181 voxels and the segmentation results of each image, including white matter (WM), gray matter (GM) and cerebrospinal fluid (CSF), are provided by BrainWeb. One of the images was selected as the template image, and others were used as subject images. To visualize the registration accuracy, the same cross section of the average brain images reconstructed from the warped subject images obtained by using different approaches are shown in Figure 2. The same cross section of the template image is also included in Figure 2a for reference. It is observed from Figure 2 that the average brain image obtained by using the proposed method is most similar to the template image than those obtained via other methods. The tissue overlap measure proposed in [1] was also adopted to quantitatively evaluate the registration accuracy of different methods. It is defined as $P=\frac{N(A \cap B)}{N(A \cup B)}$, where $A$ and $B$ denote the regions of a specific tissue in two images. The mean values and SDs of $P$ for WM, GM and CSF are listed in Table 1 for various methods. It is observed that the proposed ECF feature achieves the highest value of $P$ among all the compared methods. The average computation times for FFD, Demons, MDGM, HAMMER and ECF are 4.8hr, 2.6hr, 18.5hr, 5.4hr and $5.2 \mathrm{hr}$, respectively.

Then, we evaluated the proposed method on the IBSR dataset with all twelve brain MR images. Each image has size of $256 \times 256 \times 128$ voxels. The expert segmentation results of WM, GM and CSF are provided by IBSR. The ex-

\footnotetext{
${ }^{2}$ http://www.cma.mgh.harvard.edu/ibsr/

${ }^{3}$ http://brainsuite.usc.edu/

${ }^{4}$ http://www.itk.org/

${ }^{5}$ https://www.rad.upenn.edu/sbia/software/index.html
} 


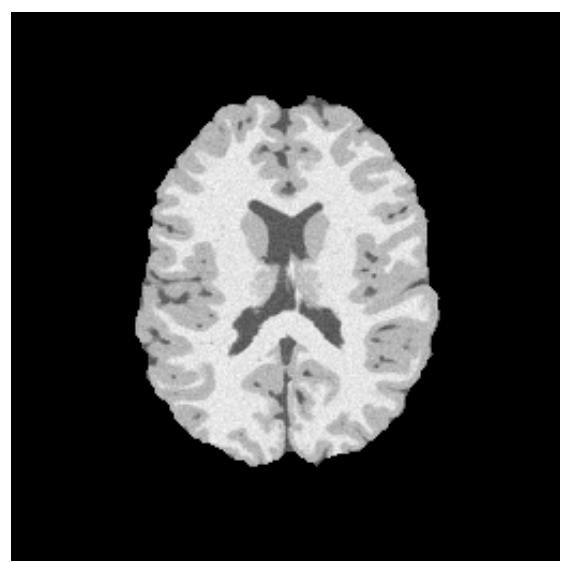

(a) Template

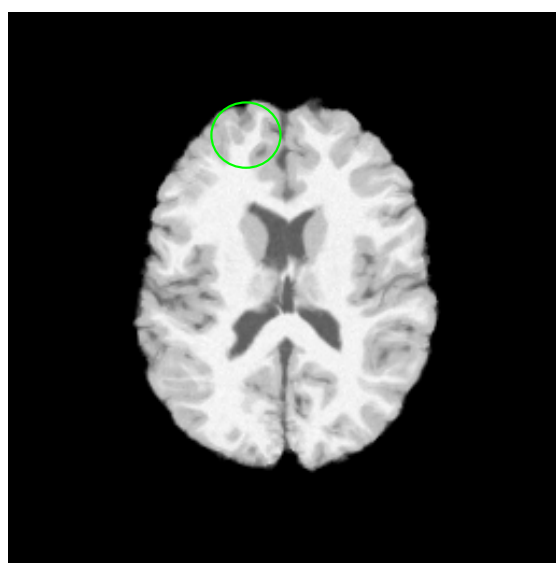

(d) HAMMER

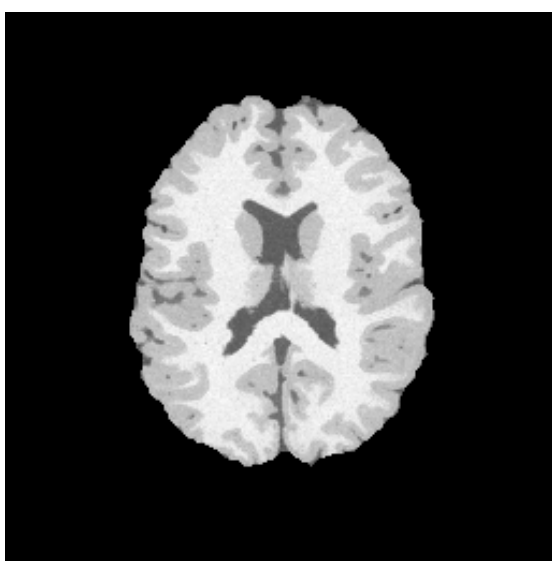

(b) Our Method

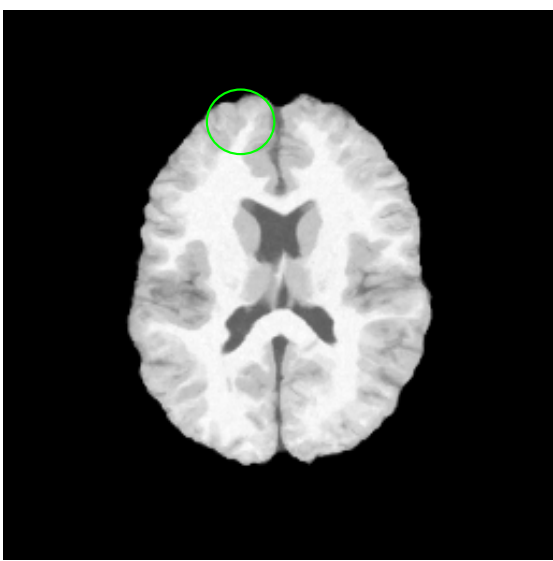

(e) FFD

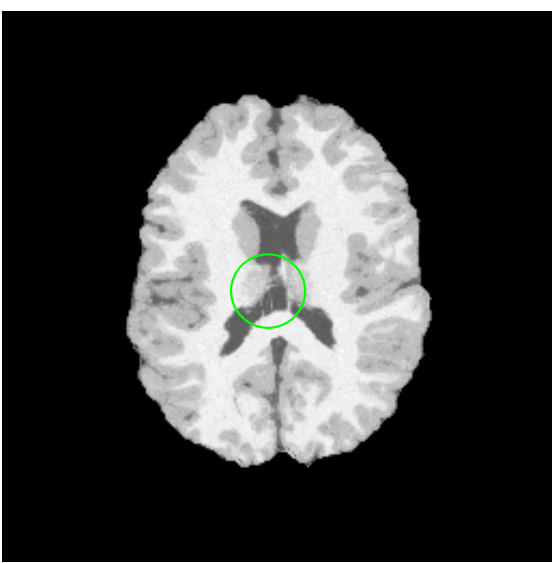

(c) MDGM

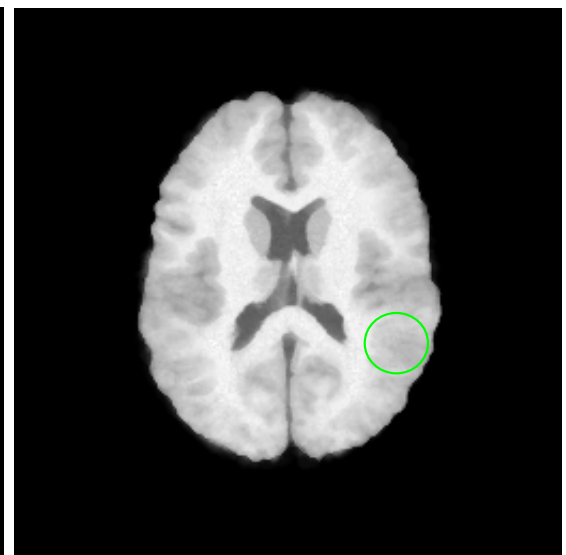

(f) Demons

Figure 2. (a) The template image; From (b) to (f): The average brain images obtained by using the proposed method, MDGM [2], HAMMER [6], FFD [5] and Demons [8] respectively for the BrainWeb database. Regions with significant differences are highlighted with green circles. Note that the skulls of input images had already been removed by using the Brainsuite software.

\begin{tabular}{|c|c|c|c|c|c|c|}
\hline Tissue & BR & FFD & Demons & MDGM & HAMMER & ECF \\
\hline Gray & $0.402 \pm 0.04$ & $0.771 \pm 0.03$ & $0.782 \pm 0.06$ & $0.696 \pm 0.03$ & $0.814 \pm 0.06$ & $0.824 \pm 0.04$ \\
White & $0.462 \pm 0.07$ & $0.748 \pm 0.04$ & $0.760 \pm 0.08$ & $0.712 \pm 0.06$ & $0.807 \pm 0.05$ & $0.811 \pm 0.06$ \\
CSF & $0.393 \pm 0.05$ & $0.737 \pm 0.07$ & $0.755 \pm 0.05$ & $0.701 \pm 0.02$ & $0.785 \pm 0.03$ & $0.807 \pm 0.01$ \\
\hline
\end{tabular}

Table 1 . The mean values of $P$ and SDs of GM, WM and CSF with different methods on the simulated 3D database. $B R$ denotes before registration.

periment setting is similar to the setting in the experiments on BrainWeb datasets. The means and SDs of $P$ are listed in Table 2 for various methods. It is observed that the proposed method consistently achieves the highest value of $P$ among all the compared methods, and the robustness of the proposed method is strongly implied. The average computation times for FFD, Demons, MDGM, HAMMER and ECF are $3.1 \mathrm{hr}, 1.5 \mathrm{hr}, 13.7 \mathrm{hr}, 4.6 \mathrm{hr}$ and $3.9 \mathrm{hr}$, respectively. It should be noted that the MDGM feature [2] does not give very good registration accuracies on both the BrainWeb and
IBSR datasets, which reflects the importance of extracting anatomical features from input images with multiple scales.

\section{Conclusion}

A feature, namely Electric Current Flow (ECF), is proposed for feature based non-rigid image registration. The ECF feature aims at modeling the inter-voxel relationship as the electric current flow. It is demonstrated that both the conventional gradient magnitude and the recently proposed maximum distance gradient magnitude feature are the spe- 


\begin{tabular}{|c|c|c|c|c|c|c|}
\hline Tissue & BR & FFD & Demons & MDGM & HAMMER & ECF \\
\hline Gray & $0.427 \pm 0.03$ & $0.724 \pm 0.02$ & $0.748 \pm 0.08$ & $0.657 \pm 0.03$ & $0.771 \pm 0.06$ & $0.796 \pm 0.06$ \\
White & $0.464 \pm 0.04$ & $0.708 \pm 0.04$ & $0.735 \pm 0.03$ & $0.638 \pm 0.05$ & $0.783 \pm 0.05$ & $0.791 \pm 0.05$ \\
CSF & $0.403 \pm 0.03$ & $0.723 \pm 0.06$ & $0.703 \pm 0.02$ & $0.604 \pm 0.05$ & $0.765 \pm 0.03$ & $0.783 \pm 0.06$ \\
\hline
\end{tabular}

Table 2. The mean values of $P$ and SDs of GM, WM and CSF with different methods on the real 3D database. BR denotes before registration.

cial cases of the proposed ECF feature. The ECF features are integrated with the Markov random field based deformation model to drive the non-rigid registration process. The proposed method has been compared with four related registration approaches (FFD, Demons, MDGM and HAMMER) on both the simulated and real 3D image databases. It is shown that the proposed method consistently achieves the highest registration accuracies among all the compared methods and its computation efficiency is comparable.

\section{Acknowledgment}

The authors would like to acknowledge the generous support from the K. S. Lo Foundation and Hong Kong GRF grant 612308 .

\section{References}

[1] W. Crum, D. Rueckert, M. Jenkinson, D. Kennedy, and M. Smith. A framework for detailed objective comparison of non-rigid registration algorithms in neuroimaging. In $M I C$ CAI, pages 679-686, 2004.

[2] R. Gan, A. Chung, and S. Liao. Maximum distance-gradient for robust image registration. MedIA, 12:452-468, 2008.

[3] B. Glocker, N. Komodakis, N. Paragios, G. Tziritas, and N. Navab. Inter and intra-modal deformable registration: Continuous deformations meet efficient optimal linear programming. In IPMI, pages 408-420, 2007.

[4] J. Pluim, J. Maintz, and M. Viergever. Image registration by maximization of combined mutual information and gradient information. TMI, 19:809-814, 2000.

[5] D. Rueckert, L. Sonoda, C. Hayes, D. Hill, M. Leach, and D. Hawkes. Nonrigid registration using free-form deformations: application to breast $\mathrm{mr}$ images. TMI, 18:712-721, 1999.

[6] D. Shen and C. Davatzikos. Hammer: hierarchical attribute matching mechanism for elastic registration. TMI, 21:14211439, 2002.

[7] W. Tang and A. Chung. Non-rigid image registration using graph-cuts. In MICCAI, pages 916-924, 2007.

[8] J. Thirion. Image matching as a diffusion process: an analogy with maxwell's demons. Med. Image Anal., 3:243-260, 1998.

[9] Z. Tu, K. Narr, P. Dollar, I. Dinov, P. Thompson, and A. Toga. Brain anatomical structure segmentation by hybrid discriminative/generative models. TMI, 27:495-508, 2007.

[10] G. Wu, F. Qi, and D. Shen. Learning-based deformable registration of mr brain images. TMI, 25:1145-1157, 2006.
[11] B. Yuri, V. Olga, and Z. Ramin. Fast approximate energy minimization via graph cuts. PAMI, 23:1222-1239, 2001. 